\title{
Entre la renovación y la reevaluación. Jalones en la historiografía francesa sobre la Restauración ${ }^{1}$ Between Renewal and Reevaluation. Milestones in French Historiography on the Restoration
}

\author{
Jean-Claude Caron \\ Université Blaise Pascal, Clermont-Ferrand
}

Recibido: 22-VII-2014

Aceptado: 1-X-2014

\section{Resumen}

La historiografía francesa sobre la Restauración está marcada por algunos importantes jalones. Poco a poco ha ido escapando de la escritura militante para convertirse en un objeto histórico sometido a una mirada crítica. Aunque durante mucho tiempo fue un período casi abandonado, progresivamente ha ido suscitando mayor interés, algo que puede apreciarse en la ampliación de las temáticas y en el peso creciente de la historia cultural que se infiltra en la historia política, social o religiosa. A pesar de ello, la historia económica o la historia comparada de las Restauraciones en Europa se encuentran todavía en un estado embrionario, a pesar de algunas iniciativas recientemente puestas en marcha.

Palabras clave: Restauración, historiografía, síntesis, cronología, ruptura, periodización, transición, diversificación temática, reevaluación histórica, arcaísmo, modernidad, construcción nacional.

1. El corpus de obras utilizadas se limita a una sesentena de referencias. Está fundado, en parte, sobre la subjetividad, pero no pretende establecer ninguna jerarquía entre las obras citadas y aquellas que no figuran aquí. Está también construido sobre obras dedicadas solamente a la Restauración, y de otras que la incluyen en cronologías más amplias. No han sido tenidos en cuenta los artículos de revista, los diccionarios históricos ni las biografías. En este último caso, la abundancia de títulos era imposible de gestionar en el marco de este artículo. Tampoco se han incluido las tesis todavía sin publicar. Para no hacer demasiado pesadas las notas al pie de la página hemos situado al final del artículo la lista de las obras citadas, referenciadas in extenso, mientras que las notas al pie del artículo se reducen a dar cuenta del autor y año de publicación. 


\begin{abstract}
French historiography of the Restoration is marked by some important milestones. It has gradually abandoned militant writing to become a historic object submitted to a critical view. Despite having been for a long time considered as a nearly forgotten period, it has of late aroused a renewed interest which is made evident by the extension of themes, and by the increasing weight of cultural history which penetrates political, social and religious history. However, the economic history, as well as the compared history of the Restorations in Europe still remains embryonic, in spite of some recent initiatives.
\end{abstract}

Keywords: The Restoration, historiography, synthesis, chronology, break- sequenced division, transition, thematic diversification, historic revaluation, archaism, modernity, national construction.

La historiografía francesa sobre la Restauración ha sido, durante mucho tiempo, reveladora de una cuestión que puede constatarse también para otros períodos: el campo de la investigación histórica no escapa al juego de la política que, con frecuencia, se manifiesta muy presente y puede llegar a ser opresiva. ¿Es necesario emanciparse? La respuesta es que, por lo menos, conviene ser consciente de ello. No es difamatorio afirmar que, en concreto durante la Guerra Fría -pero la situación se verifica también en épocas anteriores como la Tercera República-, la historia era una disciplina ampliamente instrumentalizada por las ideologías de todo tipo. Pero, como el objeto de este artículo es la etapa de la Restauración, fue precisamente durante este período cuando la historiografía, en el auténtico sentido de la palabra, adoptó una dimensión política nueva. En relación con la guerra ideológica y política que, prolongando por otros medios los enfrentamientos del período revolucionario pretendía imponer una lectura dominante de este pasado próximo, los historiadores no escaparon de la adhesión partidaria al régimen, suponiendo que en algún caso hubieran querido sustraerse a ella. Agitando un programa semejante, la palabra "restauración" se opone a la, no menos programática, de "revolución". Los trabajos de Adolphe Thiers o los de François Mignet intentaron entonces rehabilitar en bloque lo que los teóricos contrarrevolucionarios como el abate Barruel, Joseph de Maistre, Louis de Bonald condenaban, también ellos, en bloque.

El triunfo de los liberales en 1830 durante las Tres Gloriosas se tradujo en la consolidación de una "leyenda negra de la Restauración" de la que participan, especialmente, Achille de Vaulabelle o Louis Blanc. Una legenda negra que reforzará la historiografía republicana, comenzando por Jules Michelet, haciendo de la Restauración, y pronto de la Monarquía de julio, un simple paréntesis en la historia. En el campo de los legitimistas, Jean-Baptiste Capefigue, y después Alfred Nettement, se erigieron en defensores de la Restauración. Prototipo de alto funcionario servidor del Estado y de la República, Sébastien Charléty 
trata del periodo en "la" Lavisse, esa Histoire de la France contemporaine que constituye un monumento de relato nacional de la Revolución hasta el día siguiente de la Primera Guerra Mundial. En el seno de la historia socialista dirigida por Jean Jaurès, es René Viviani, cofundador con este último de L'Humanité, quien da forma al período. Bajo la República triunfante, la historia de la Restauración se encuentra en manos de historiadores que no dudan en postularse como procuradores o como abogados del período y del régimen político que la caracteriza, la monarquía constitucional, acentuando de hecho el estereotipo de la "Restauración-reacción". Pero, como lo resume Martine Reid (2005), todavía en el tiempo presente, "la Restauración estrangulada entre el Imperio, rápidamente paso rápidamente a formar parte del conjunto de los grandes mitos nacionales, y la monarquía de Julio que verá el triunfo del liberalismo, parece decididamente condenado a figurar en el catálogo de manifestaciones de "otro tiempo""'. No obstante, el balance reciente de la historiografía de la Restauración tiende a desmentir un poco esta afirmación, que es lo que nosotros nos hemos propuesto demostrar. Ahora es posible releer el período, como invitan a hacerlo los coordinadores de un número de la Revue d'histoire du XIXe siècle consagrado a la "Restauración revisitada" (Christen-Lécuyer et Fureix, 2007), considerando el período bajo el triple ángulo de la transacción, de la recomposición y de la nostalgia ${ }^{3}$.

\section{Algunos jalones historiográficos: manuales y obras de síntesis}

Autor de la síntesis culta de referencia sobre la Restauración, el padre Guillaume de Bertier de Sauvigny (1955) se inscribe en una tradición que podría ser calificada de historia militante. Este autor abre así su estudio: "1814... Francia exhausta después de veinte años de fiebre revolucionaria y conquistadora sufre el enfrentamiento con las mismas fuerzas que había desencadenado" y su conclusión refuerza el planteamiento inicial. "Hemos sido llevados fatalmente a preguntarnos, después de concluida, si la Restauración era un régimen viable para Francia. La Revolución de 1830 parece, a primera vista, demostrar que no. Este es un mal argumento, porque esta revolución, se ha demostrado, fue un accidente perfectamente evitable ${ }^{4 "}$. En cuanto al balance de la Restauración, es poco decir que Guillaume de Bertier de Sauvigni la encuentra globalmente positiva, como otros lo decían entonces del régimen soviético: "Nunca, sin duda,

2. ReID, Martine, «Introduction», en MOLlier, ReID y Yon (2005), p. 8.

3. Christen-LéCuyer, Carole y Fureix, Emmanuel (2007), p. 10.

4. Bertier de SAUvigny, Guillaume de, La Restauration, Flammarion, 1974 (1a edic. 1955), pp. 9 y 460-462. El subrayado es nuestro. 
Francia ha estado mejor administrada, con más honestidad, desde arriba hasta abajo de la escala jerárquica, con más respeto por las leyes y los reglamentos, con más miramientos hacia los derechos de los ciudadanos, con menos abusos en el uso de los recursos públicos" ${ }^{5}$. El éxito de este estudio (reeditado en 1963 y en $1974^{6}$, traducido al ingles en $1966^{7}$, reimpreso hasta en 1998) se explica por sus incontestables calidades científicas, pedagógicas y literarias. Pero en realidad ocupaba un vacío historiográfico que solo irá siendo cubierto muy lentamente.

Si intentamos analizar las razones de lo que sería una renovación de la historia del período, podríamos sugerir que los debates historiográficos despertados por el bicentenario de la Revolución francesa en 1989 tuvieron también su impacto sobre la percepción de la Restauración, tanto que esta fue regularmente convocada en los coloquios o publicaciones para captar la herencia de la Revolución francesa entre 1814 y 1830, tuvo su lugar en los combates políticos del período e, incluso, en la apuesta por la escritura de una "historia en caliente" que ve, precisamente, la luz en este momento ${ }^{8}$. Surge también la voluntad de ciertos historiadores, comenzando por Alain Corbin, de imponer una lectura menos "cívica" o teleológica de los años 1815-1871 -por lo tanto, también de la Restauración- en beneficio de una aproximación "sensible" fundada sobre el estudio de fuentes hasta entonces poco utilizadas; de reevaluar el peso de las emociones populares, en particular las rurales; de mostrar la complejidad de la formas de creencia, de adhesión o de rechazo del discurso de las élites; y también de recurrir a introducir conceptos etnológicos o antropológicos en la "caja de herramientas" de un tipo de historiador singularmente amplio.

A pesar de ser obras de circunstancia o alimenticias, los manuales y obras de síntesis ofrecen una imagen muy fiable de la evolución historiográfica de un país. Todo esto cobra sentido, desde la cronología contemplada hasta las lagunas, ya sean reivindicadas o no. Todas las colecciones históricas dedicadas a la historia contemporánea han tomado en consideración a la Restauración. Es el caso de la célebre colección "Que sais-je?", publicada por Presses Universitaires de France, que recurre a Jean Vidalnc (1966), y después a Jean-Pierre Chaline (1998), o de la Nouvelle histoire de la France contemporaine publicada por Seuil que, sin embargo, trata como un bloque la monarquía constitucional (1814-1848). Los dos volúmenes de la colección firmados por André Jardin

5. Idem, p. 461.

6. Bajo el título: Au soir de la monarchie. La Restauration, Paris, Flammarion, 1974.

7. The Bourbon Restoration, Philadelphia, The University of Pennsylvania Press, 1966.

8. Voir Bourdin, Philippe (dir.), La Révolution, 1789-1871. Ecriture d’une histoire immédiate, Clermont-Ferrand, PUBP y Vizille, Musée de la Révolution, 2008. 
y André-Jean Tudesq (1973) constituyeron un jalón historiográfico importante, si tenemos en cuenta su amplia y duradera difusión, pero también de su título que subrayaba la primacía de un grupo, La France des notables, una expresión que se ha convertido en lugar común para designar el período. La versión reactualizada, obra de Bertrand Goujon, muestra a través de su titulo, Monarchies postrévolutionnaires (2012), su voluntad de inscribir la época en estrecha relación con la Revolución francesa. Todavía más sobrio parece el título del volumen redactado por Jean-Claude Caron, La France de 1815 a 1848 (1993), editado por Armand Colin en la colección Cursus. La secuencia abordada en los volúmenes dirigidos por François Furet (1988), Dominique Barjot, Jean-Pierre Chaline y André Encrevé (1995) o Sylvie Aprile (2010) es mucho más amplia: 1770-1880 en el primer caso, 1814-1914 en el segundo, 1815-1871 en el tercero.

Específicamente dedicadas a la Restauración, algunas síntesis recientes han renovado la percepción del período. Es el caso de la Histoire de la Restauration publicada por Emmanuel de Waresquiel y Benoît Yvert en 1996, cuyo subtítulo "El nacimiento de la Francia moderna" aparece como una suerte de manifiesto, rechazando la visión de un período caracterizado por su arcaísmo. Lo mismo sucede con La France de la Restauración, de Francis Démier aparecido en 2012, cuyo subtítulo, "El imposible retorno al pasado", pone de manifiesto la voluntad de cuestionar la habitual representación del régimen poniendo el acento sobre las tensiones ideológicas y políticas del período. ¿Qué nos dicen estos manuales y estas síntesis? En primer lugar, que la interpretación del régimen político de la Restauración en términos jurídico-constitucionales es un debate en sí mismo. Si el acuerdo es casi unánime en lo referente a que el período no es una monarquía parlamentaria -los historiadores franceses reservan la expresión para el régimen orleanista, no sin reservas muchas veces-, los puntos de vista divergen entre "monarquía constitucional" (Goujon, 2012), "monarquía limitada" (Aprile, 210) o "a medio camino entre monarquía absoluta y monarquía parlamentaria" (Caron, 1993). Estas síntesis revelan igualmente el peso persistente de la historia política y social, combinado, sin embargo, con una caída de los trabajos de historia regional que habían inspirado tantas tesis durante los años 1950-1980, y el ascenso de una historia cultural ampliada a una dimensión etnoantropológica. Pero también ponen de manifiesto las ausencias o las lagunas de la historiografía, en primer lugar de las cuales debe señalarse la historia económica, además de la historia comparada, internacional o diplomática. Por mostrar un ejemplo, la noción de Santa Alianza ha sido muy poco estudiada recientemente. Y si la historia de la producción escrita, sea cual sea su género (prensa, novela, teatro, historia, etc.) ha producido trabajos mayores, estos se inscriben raramente en una dimensión comparativa. 


\section{Variaciones cronológicas}

Las secuencias cronológicas constituyen una apropiación de la historia y revelan la intención de sus autores. Durante mucho tiempo, la Restauración ha sido estudiada de dos maneras. La primera proponía una especie de "imagen detenida" como hace Henri Houssaye (1848-1911), historiador de la Belle Époque, reduciendo la Restauración a un tiempo corto, el que se extiende entre 1814 y 1815. La tetralogía que forman su 1814 y los tres volúmenes de su $1815^{9}$ fueron auténticos best-sellers: se vendieron más de 75.000 ejemplares antes de 1914 , y continuaron su carrera en el período de entre guerras hasta superar las 90 ediciones en los años 30. Recientemente, algunos historiadores han reconsiderado el interés de este tiempo corto. Es el caso de Laurent Nagy (2012) que explora los complots bonapartistas para los años 1815-1816 o Sébastien Allard (2005) interrogando a la generación romántica de 1820.

La segunda considera a la Restauración como si fuera un bloque que abarca los años 1814/15-1830 compuesto por elementos internos muy compartimentados. El año 1814-1815, desde la primera a la segunda abdicación de Napoleón, es en sí mismo una secuencia que, desde muchos puntos de vista, aparece como un "año terrible" avant l'heure, para parafrasear a Victor Hugo en la caracterización que hizo del año 1870-1871. El período que va desde junio de 1815 hasta febrero de 1820 es estudiado para analizar el combate que se produce entre una concepción más o menos abierta o restrictiva de los derechos afirmados por la Carta, y se cierra con el asesinato del duque de Berry. Los años 1820-1827, marcados por la personalidad del duque de Richelieu, y todavía más por Villèle, están caracterizados por el retorno hacia un tradicionalismo político que se acentúa con la llegada de Carlos X al trono de Francia. Pero son los años siguientes, desde el éxito de los liberales en las elecciones del otoño de 1827 a la revolución de julio de 1830, los que dan testimonio de una evolución tanto más interesante en la medida que se inserta sobre un debate historiográfico de primera magnitud: la temporalidad y las modalidades de la transición entre dos regímenes políticos. Sobre este punto, la articulación Restauración-monarquía de julio constituye un caso único en la historia contemporánea de Francia, conservando la misma constitución después de la revolución tras realizar, simplemente, algunos arreglos.

Se entiende así el interés de la secuencia 1828-1832 adoptada por Bertrand Goujon (2012) quien relativiza de facto la ruptura que habrían significado las Tres Gloriosas. Más todavía, es la propia temporalidad de la revolución, más o menos dilatada, la que es puesta en cuestión: tres jornadas revolucionarias,

9. HoussaYe, Henry, 1814, Paris, Perrin, 1888; 1815, Paris, Perrin, 1893-1905, 3 vols. 
cierto, pero ¿quien queda arriba y quien abajo? El autor responde a esto fragmentando el quinquenio 1828-1832 en finas láminas cronológico-políticas donde se suceden "la moderación constitucional, la reacción realista, la revolución, la alternancia dinástica, la apertura a la izquierda y la recentralización conservadora ${ }^{10}$ ". Haciendo esto, Bertrand Goujon sigue los pasos de Jean Bruhat (1979) quien, en el seno de la Historie de la France contemporaine impulsada por algunos historiadores comunistas, trataba como un solo bloque los años 1827-1835, ampliando todavía más la secuencia de transición entre las elecciones del otoño de 1827, marcadas por la aparición de las barricadas en París, y las leyes sobre la prensa de septiembre de $1835^{11}$. En este sentido, deberíamos citar también a Maïte Bouyssy (2012) quien identifica un "momento frenético francés" entre 1824 y 1834, que aspira a resumir en tres palabras: la urgencia, el horror y la democracia. Por nuestra parte, la incertidumbre que prevalece al comienzo de la monarquía de julio, un factor insuficientemente valorado por los historiadores, nos impulsó a recuperar una expresión contemporánea de los años 1830-1832, "la época sin nombre" (Caron, 1993). En consecuencia, lo que se hizo para el final de la Restauración queda todavía pendiente de hacer para sus inicios: la idea de ruptura neta y radical con el Primer Imperio continua dominando la historiografía, de ahí la concomitancia entre la invasión extranjera de Francia en dos ocasiones, la caída de Napoleón y, más allá del final de su régimen, el de la secuencia revolucionaria iniciada en 1789. Sin embargo, la transición entre Primer Imperio y Restauración merecería ser revisitada en la media duración. Por ejemplo, para el período que va desde la campaña de Rusia y de sus efectos sobre la opinión pública hasta el asesinato del duque de Berry en 1820, que abre un período de reacción antiliberal muy pronunciado.

Dicho esto, numerosas obras incluyen la Restauración en una secuencia cronológica más amplia que los historiadores franceses han venido generalmente denominando "primer siglo XIX", expresión que designa generalmente los años 1815-1871, incluso, de manera más restringida los años 1814-1848 (KarilaCohen, 2008) o 1814-1851 (Caron, 1991). Como excepción, Emmanuel Fureix (2009) ha propuesto una secuencia 1814-1840 adaptada a su objeto de estudio: los funerales políticos. El trayecto de integración de la Restauración en un tiempo más largo es a la vez significativo y estimulante. Significativo porque, salvo raras excepciones, está siempre integrada al después, ese "primer siglo XIX" del que constituye el inicio, y no al antes, al período de la Revolución francesa-

10. Goujon, Bertrand (2012), p. 203.

11. Elleinstein, Jean (dir.), Histoire de la France contemporaine, t. 2, 1799-1835, Editions sociales - Livre Club Diderot, 1979. 
Primer Imperio. Solo puede señalarse aquí el carácter pionero del estudio dedicado por Maurice Agulhon (1977) al Cercle dans la France bourgeoise en los años 1810-1848 y la originalidad de la secuencia tratada por François Ploux (2002), los años 1810-1860, o Aurélien Lignereux (2008), los años 1800-1859, aunque señalando que se trata sobre todo de excepciones. Esto sugiere, por lo tanto, que la idea de ruptura que comporta la Restauración se encuentra fuertemente anclada en la cronología, como demuestra igualmente la periodización que continua prevaleciendo en la enseñanza universitaria. Su integración en ese "primer siglo XIX" se demuestra muchas veces estimulante porque, no sin el riesgo de una lectura teleológica del pasado, sitúa a la Restauración como el origen del funcionamiento de instituciones que, si bien no son parlamentarias, reconocen las nociones de derechos garantizados, de igualdad jurídica, de representación nacional y de aceptación de la ley o del impuesto, todas ellas nociones que anuncian la democracia parlamentaria que está por venir.

\section{Diversificación temática}

La diversificación de las temáticas revela hasta qué punto la historia es sensible a renovaciones de perspectiva que traducen la evolución de fuerzas en el seno de la disciplina histórica. apoyaremos nuestra propuesta sobre un número limitado de obras, pero, desde nuestro punto de vista, muy significativas de esta mutación. Poseen un punto en común: si bien todas retoman la noción de "romanticismo" hasta el punto de incluirlo en su título, todas consideran necesario redefinir este "momento romántico" para salir de una imaginería convencional. Así, hacer de los estudiantes de París unas "generaciones románticas" (Caron, 1991) sirve ante todo para plantear cómo se construye la representación socio-cultural de un grupo sometido a diversos estereotipos políticos, literarios, iconográficos de los cuales es necesario mostrar su origen y su intención para deconstruirlos mejor. Ciertos campos aparecen menos frecuentados que en otro tiempo, como la historia política o la historia religiosa. En el primer caso, se observa frecuentemente una combinación de cerrazón temática y ampliación cronológica, al estilo del estudio de la candidatura oficial estudiada por Christophe Voilliot (2005). Pero los estudios de ideología o de corrientes políticas siguen de actualidad, tanto si se trata de los sansimonianos (Picon, 2002), de los liberales del diario Le Globe (Goblot, 1995), de los doctrinarios (Craiutu, 2006) o de la derecha en general (Tort, 2013). Y si un historiador de lo político como Pierre Rosanvallon ha dedicado un amplio espacio a la Restauración en sus trabajos sobre el sufragio (1992), la representación democrática (1998) o la soberanía del pueblo (2000), igualmente ha tomado como objeto de estudio las Cartas de 1814 y de 1830, conectando así con una tradición de historia 
constitucional abandonada (1994). Esta conducta es comparable a la de Alain Laquièze (2002) que se interesa por los orígenes del régimen parlamentario, un tema que había caído en el olvido desde hacía ya algún tiempo.

En el terreno religioso, los trabajos pioneros de Claude Langlois (1974, 1984), Michel Lagrée (1977) o Philippe Boutry $(1985,1986)$ han tenido una descendencia limitada, entre la cual puede citarse el estudio de la Grande Mission de Besançon obra de Gastón Bordet (1998) o el Mythe jésuite deconstruido por Michel Leroy (1992). La historia institucional de la Iglesia resulta bastante desatendida, en particular en lo que respecta al episodio del concordato de 1817, estudiado por Antoine Roquette (2010). Es cierto, no obstante, que historia política e historia religiosa han sido profundamente renovadas gracias a la aportación más o menos masiva de conceptos traídos de otras disciplinas como la antropología o la sociología, y apoyándose sobre la poderosa ola de la historia cultural. El estudio de los pares de Francia realizado por Emmanuel de Waresquiel (2006) o también el volumen dirigido por Matthieu Brejon de Lavergnée y Oliver Tort, consagrado a la unión del Trono y del Altar (2002), ilustran esta persistencia de una historia sociopolítica, pero cruzada de un enfoque cultural. Sucede lo mismo con el estudio de la invención de los sondeos políticos al que se entrega Pierre Karila-Cohen (2008) o también de la historia del rumor tratada por François Ploux (2003).

$¿$ Que otro volumen ofrece un testimonio mejor que Repenser la Restauration (Mollier, Reid y Yon, 2005) sobre el impacto de la historia cultural sobre la relectura de la Restauración? Los títulos de las seis partes que reagrupan veintisiete contribuciones dan testimonio de ello: "Pasado y presente de la historia"; "Ideologías"; "Políticas artísticas"; "Libro, imagen, sociedad"; "Teatro, risa y sátira"; "Restauración y romanticismo". Como puede verse, se trata de un verdadero catálogo (sin atribuir a este término ningún matiz peyorativo) de las canteras de la historia cultural que, sin embargo, no olvida tampoco la dimensión socio-política de los temas. La obra pionera de François Parent-Lardeur (1981) sobre los gabinetes de lectura bajo la Restauración ilustra este punto, interesándose al mismo tiempo por los libros, las lecturas y los lectores. Como subraya Daniel Roche, es "a través de la confrontación de lo social en el espacio urbano cuando se definen las apuestas de la institución 'gabinete de lectura': de lo político a lo cultural ${ }^{12 "}$. El único límite que se puede ver al ejercicio es, a pesar de algunas raras excepciones, privilegiar las elites o, lo que es lo mismo, las categorías sociales que, de una u otra manera, tienen acceso a uno o varios

12. Roche, Daniel, compte rendu de Françoise Parent-Lardeur (1981), en Annales. Économies, sociétés, civilisations, , vol. 39, nº 1 (1984), p. 183. 
modos de expresión: filosofía, historia, periodismo, escritura de ficción, formas artísticas diversas, derecho de elección o de elegibilidad, etc., en resumen, las capacidades sancionadas por un diploma (Caron, 1991; Yannick Le Marec, 2000) o, por ejemplo, las élites integradas en el mundo de la filantropía (Duprat, 1996-1997). ¿Quiere esto decir que la Restauración constituye un caso a parte en este tema? Emmanuel de Waresquiel responde a su manera (2005) demostrando que las élites se encontraban entonces en el corazón de una producción discursiva mayor en relación con las apuestas sociales y políticas que caracterizan el período. Se puede, por otro lado, unir esta cuestión a la de las apuestas memoriales de la Revolución y del Imperio que estudia Natalie Petiteau (2012) o a la de las memorias cuya enorme producción bajo la Restauración ha sido señalada por Damien Zanone (2006).

En cuanto al resto, las fronteras entre géneros o categoría históricas se difuminan cada vez más como pone de manifiesto el estudio de los banquetes al que se ha dedicado Vincent Robert (2010), con una lectura de su objeto a la vez político, social y cultural. Y, aunque es difícil establecer los límites de la historia cultural, hay una evidencia que está fuera de toda duda: en grados diversos, se ha impuesto en la relectura el período, sea de manera autónoma, o de manera complementaria con otro tipo de aproximaciones. Ya se trate de la canción política y social -Darriulat, 2010- o de los discursos laudatorios a la gloria de los Borbones restaurados -Legoy, 2010-, se ha exhumado una gran parte de la producción literaria que ha dejado de considerarse como una mirada anecdótica o ilustrativa para ser interrogada sobre la representación que puede ofrecer del régimen de la Restauración. El registro de lo afectivo, del pathos o de la emoción es también convocada: se encuentra en La France des larmes de Emmanuel Fureix (2009), cuyo subtítulo, Deuils politiques à l'âge romantique indica que se trata -fundamentalmente, pero no exclusivamente- de una historia de lo emotivo, tan valorada por Alain Corbin. Se encuentra esta influencia en un terreno que ha conocido y conoce un importante desarrollo desde hace una veintena de años: el de la violencia o de las violencia, sean cuales sean la naturaleza y el productor. Este tema aflora en las obras de Maite Bouyssy (2012), François Jarrige (2009) o François Ploux (2002). Constituye el corazón de Les Grains du désordre, en el cual Nicolas Bourguinat (2001) estudia las respuestas del Estado ante las violencias por el trigo (2001), en los Feux de la discorde de Jean-Claude Caron (2006), que trata del recurso al incendio en los conflictos políticos o privados, o también en France rébellionnaire d'Aurélien Lignereux (2008), que abarca en todo el ámbito nacional la amplia cuestión de las resistencia a la gendarmería. Alimenta también la obra que Gilles Malandain (2011) ha dedicado a Louvel, el asesino del duque de Berry, renovando de este modo la cuestión del regicidio. 


\section{¿Una reevaluación histórica?}

Aunque intentar una rehabilitación de la Restauración no está en el punto de mira, lo que sí puede decirse es que existe una reevaluación en nombre de un necesario y saludable revisionismo histórico. Muchos de los historiadores del periodo justifican su empresa por la propia constatación de un hecho: entre el desinterés de los investigadores y la persistencia de una leyenda negra, la Restauración debe ser reevaluada, aunque solo sea para poner en evidencia las paradojas, incluso las contradicciones de un período que se debate entre dos tendencias que podrían ser denominadas como arcaísmo y modernidad. Es esta tensión manifiesta, permanente y productiva (de palabras y de actos) la que constituye la originalidad de un momento que hace frente a un "pasado que no pasa", por retomar la fórmula aplicada por Éric Conan y Henry Rousso a la Francia de Vichy ${ }^{13}$, pero también, y tal vez sobre todo, a un futuro repleto de incertidumbre.

Siguiendo los pasos de Pierre Rosanvallon, Pierre Karila-Cohen sugiere que los años 1814-1848 constituyen un "laboratorio político" de donde emerge "una racionalidad nueva, que hace de la opinión el horizonte de la acción gubernamental ${ }^{14 "}$, unido en esto a Emmanuel Fureix que define este momento político como "el de una reflexión nueva sobre la opinión pública, entendida como esfera pública burguesa, y de una inquietud sobre los 'murmullos' populares y la pluralidad de las opiniones ${ }^{15}$ ". Haciendo esto, el uno y el otro asumen estar realizando una reevaluación de la jerarquía del discurso. De la confrontación de fuentes aparentemente sin conexión surge la necesidad de una renovación metodológica, tomando en cuenta, por ejemplo, la noción de imaginario social. Paralelamente, Maïte Bouyssy invita a una lectura dinámica de las fuentes constatando, a partir de los trabajos de Alberto M. Banti, que "las cuadrículas de lectura se adaptan, se entrecruzan, se recortan, porque no existe herencia cultural que se transmita y no se trasforme ${ }^{16 "}$.

Hay, sin embargo, lagunas temáticas persistentes en la producción histórica sobre la Restauración. Al final de su imponente análisis (más de 950 páginas), Francis Démier muestra hasta qué punto lo económico, frecuentemente dejado de lado, y lo social proporcionan una plantilla de lectura esencial para comprender un régimen frecuentemente percibido prioritariamente, incluso exclusivamente, desde una perspectiva ideológica, política o cultural. Su propósito

13. Conan, Éric et Rousso, Henry, Vichy, un passé qui ne passe pas, Paris, Fayard, 1994.

14. Karila-Cohen, Pierre (2008), p. 13.

15. FUREIX, Emmanuel (2009), pp. 16-17.

16. BouYssy, Maïté (2012), p. 290. 
es un alegato en favor de lo socioeconómico como factor determinante, en una época donde un pequeño número de hombres, encarnación de una ciudadanía electoral muy limitada en un régimen estrictamente censitario, son en primer lugar productores, emprendedores o rentistas también, cuyo poder es inversamente proporcional a su número. Pero, es evidente, que este campo es poco frecuentado por los historiadores de la Restauración, igual que lo era hasta hace muy poco tiempo el de la historia militar. En este terreno, no se trata, o no solamente, de producir una historia-batalla, sino más de una historia social, política y cultural de los soldados de la Grande Armée desmovilizados (Petiteau, 2003), de los oficiales napoleónicos caídos en busca de causas que defender (Bruyère-Ostells, 2009), de los ejércitos de ocupación (Hantraye, 2005), incluso de los voluntarios que se comprometieron al lado de los movimientos de liberación nacional, como el movimiento filoheleno (Mazurel, 2013).

¿Cuál es, en definitiva, el verdadero pariente pobre de esta historiografía? Como se habrá visto en la lectura de las líneas precedentes, la producción histórica francesa reciente se encierra frecuentemente en la perspectiva franco-francesa. Existen dos explicaciones a esto. La primera es de orden cronológico: a priori, Francia constituye una suerte de excepción europea, a la vez en la fecha de origen y en la fecha de terminación del período llamado Restauración. ¿Qué otra nación -aceptando que exista como tal- se inscribe en la cronología 1814/ 1815-1830 y en el tipo de acontecimientos -con la guerra por un lado y la revolución por otro- que justifique estos límites? La segunda razón, que se deriva de la primera, es de orden historiográfico: vista desde Francia, la Restauración no cobra realmente sentido más que en una secuencia centenario que vaya desde los años 1789 (la Ilustración) hasta los años 1880 (triunfo de la "República republicana"). Se ha tenido la oportunidad de decir aquí cuantos de estos cortes cronológicos eran a la vez impuestos y pesados en la lectura del período, pero continúan todavía siendo utilizados haciendo, de facto, muy difícil la comparación internacional porque "aíslan" en cierta forma a Francia del resto de Europa.

En consecuencia, conviene reflexionar sobre la divergencia de las temporalidades, como propone la obra colectiva dedicada a las revoluciones de 1830 en Europa (Aprile, Caron y Fureix, 2013). Inscribiendo el "momento 1830" en una secuencia ampliada, los diferentes autores proponen una confrontación de las temporalidades, pero también de los conceptos (revolución), de las categorías de actores y de las políticas de Estado para elevar un panorama tan ancho como fuera posible de una Europa de las Restauraciones englobando los años 1810, 1820 y 1830. También desde esta perspectiva han sido concebidos los dos coloquios internacionales organizados en octubre de 2013 en Clermont-Ferrand 
y en junio de 2014 en París por Jean-Claude Caron y Jean-Philippe Luis, catedráticos en la universidad Blaise-Pascal de Clermont-Ferrand, bajo el título común de Rien appris, rien oublié? Les Restaurations dans l'Europe postnapoléonienne (1814-1830). El proyecto ha consistido en confrontar las historiografías (Alemania, Bélgica, España, Francia, Países Bajos, Reino Unido, Rusia y Suiza), con la intención de encontrar tanto puntos de convergencia como de divergencia, extraer temáticas transversales y particularismos nacionales. Se trata en una primera etapa de revisitar las Restauraciones como un momento de "modernización política y económica": la expresión puede parecer paradójica porque va contracorriente de una historiografía largo tiempo fundada sobre la percepción del período como un tiempo de reacción absoluta. Pero se trata igualmente de volver sobre una lectura un poco militante de las resistencias a los regímenes restaurados, y de acotar, por ejemplo, los límites de la "internacional liberal" de los años 1820. Los campos de trabajo abiertos así se presentan tanto más prometedores cuando la internacionalización de la mirada sobre las Restauraciones en Europa se cruza con una renovación conceptual, apoyándose, por ejemplo, sobre la noción de transnacionalización. Entre diversidad de experiencias y diversidad de conceptos, la historia de las Restauraciones puede pretender igualmente una internacionalización extraeuropea interrogando la evolución de las sociedades coloniales comprometidas en el mismo momento en las guerra de independencia y en la definición de la idea de nación, como en caso de la América española. Multitud de perspectivas que demuestran, si es que hiciera falta, la actualidad de la Restauración.

Traducción al castellano a cargo de PeÑa VeróN

\section{Bibliografía de las obras citadas ${ }^{17}$}

Agulhon, Maurice, Le Cercle dans la France bourgeoise, 1810-1848. Étude d'une mutation de sociabilité, Paris, Armand Colin, 1977.

Allard, Sébastien (dir.), Paris 1820. Laffirmation de la génération romantique, Bern, Berlin et alii, Peter Lang, 2005.

Aprile, Sylvie, La Révolution inachevée, 1815-1871, Paris, Belin, 2010, coll. Histoire de France.

Aprile, Sylvie, Caron, Jean-Claude et Fureix, Emmanuel (dir.), La Liberté guidant les peuples. Les révolutions de 1830 en Europe, Seyssel, Champ Vallon, 2013.

Barjot, Dominique, Chaline, Jean-Pierre et Encrevé, André, La France au XIXe siècle, 1814-1914, Paris, PUF, 1995.

Bertier de Sauvigny, Guillaume de, La Restauration, Paris, Flammarion, 1955.

17. Solo se menciona la fecha de la primera edición. 
Bordet, Gaston, La Grande Mission de Besançon, janvier-février 1825. Une fête contre-révolutionnaire, néobaroque ou ordinaire?, Paris, Éditions du Cerf, 1998.

Bourguinat, Nicolas, Les Grains du désordre. L'É tat face aux violences frumentaires dans la première moitié du XIXe siècle, Paris, Éditions de l'EHESS, 2001.

Boutry, Philippe et Nassif, Jacques, Martin l'archange, Paris, Gallimard, 1985.

Boutry, Philippe, Prêtres et paroisses au pays du curé d'Ars, Paris, Les éditions du Cerf, 1986.

Bouyssy, Maïté, L'Urgence, l'horreur, la démocratie. Essai sur le moment frénétique français, 1824-1834, Paris, Publications de la Sorbonne, 2012.

Brejon de Lavergnée, Matthieu et Tort, Olivier (dir.), LUnion du Trône et de l'Autel ? Politique et religion sous la Restauration, Paris, Presses de l'Université Paris-Sorbonne, 2012. Bruyère-Ostells, Walter, La Grande armée de la liberté, Paris, Tallandier, 2009.

Caron, Jean-Claude, Générations romantiques. Les étudiants de Paris et le quartier latin (1814-1851), Paris, Armand Colin, 1991.

Caron, Jean-Claude, La France de 1815 à 1848, Paris, Armand Colin, 1993, coll. Cursus.

Caron, Jean-Claude, Les Feux de la discorde. Conflit et incendie dans la France du XIXe siècle, Paris, Hachette-Littérature, 2006.

Chaline, Jean-Pierre, La Restauration, Paris, PUF, 1998, coll. Que sais-je?

Christen-Lécuyer, Carole et Fureix, Emmanuel (dir.), «La Restauration revisitée», Revue d'histoire du XIXe siècle, n³5 -2007/2.

Craiutiu, Aurelian, Le centre introuvable, la pensée politique des doctrinaires sous la Restauration, Paris, Plon, 2006.

Darriulat, Philippe, La Muse du peuple. Chansons politiques et sociales en France, 18151871, Rennes, PUR, 2010.

Démier, Francis, La France de la Restauration (1814-1830). L'impossible retour du passé, Paris, Gallimard, 2012, coll. Folio histoire.

Duprat, Catherine, Usage et pratiques de la philanthropie. Pauvreté, action sociale et lien social, à Paris, au cours du premier XIXe siècle, Paris, Association pour l'étude de l'histoire de la sécurité sociale, 1996-1997, 2 vol.

Elleinstein, Jean (dir.), Histoire de la France contemporaine, t. 2, 1799-1835, Editions sociales - Livre Club Diderot, 1979.

Fureix, Emmanuel, La France des larmes. Deuils politiques à l'âge romantique (18141840), Seyssel, Champ Vallon, 2009.

Furet, François, La Révolution: de Turgot à Jules Ferry. 1770-1880, Paris, Hachette, 1988.

Goblot, Jean-Jacques, La Jeune France libérale. Le Globe et son groupe littéraire, 1824-1830, Paris, Plon, 1995

Goujon, Bertrand, Monarchies postrévolutionnaires, 1814-1848, Paris, Seuil, 2012, coll. Histoire de la France contemporaine.

Hantraye, Jacques, Les Cosaques aux Champs-Élysées. Loccupation de la France après la chute de Napoléon, Paris, Belin, 2005.

Jardin, André et Tudesq, André-Jean, La France des notables, 1815-1848, Paris, PointsSeuil, 1973, 2 vol., coll. Nouvelle histoire de la France contemporaine. 
Jarrige, François, Au temps des «tueuses de bras». Les bris de machine à l'aube de l'ère industrielle, Rennes, PUR, 2009.

Karila-Cohen, Pierre, L'État des esprits. L'invention de l'enquête politique en France (18141848), Rennes, PUR, 2008.

Lagrée, Michel, Mentalités, religion et histoire en Haute-Bretagne au XIXe siècle. Le diocèse de Rennes, 1815-1848, Paris, C. Klincksieck, 1977.

Langlois, Claude, Un diocèse breton au début du XIXe siècle. Le diocèse de Vannes, 18001830, Paris, Klincksieck, 1974.

Langlois, Claude, Le Catholicisme au féminin. Les congrégations françaises à supérieure générale au XIXe siècle, Paris, Le Cerf, 1984.

Laquièze, Alain, Les Origines du régime parlementaire en France (1814-1848), Paris, PUF, 2002.

Legoy, Corinne, L'Enthousiasme désenchanté. Éloge du pouvoir sous la Restauration, Paris, Société des études robespierristes, 2010.

Le Marec, Yannick, Les Temps des capacités. Les diplômes nantais à la conquête du pouvoir dans la ville, Paris, Belin, 2000.

Leroy, Michel, Le Mythe jésuite, de Béranger à Michelet, Paris, PUF, 1992.

Lignereux, Aurélien, La France rébellionnaire. Les résistances à la gendarmerie (18001859), Rennes, PUR, 2008.

Malandain, Gilles, L'introuvable complot. Attentat, enquête et rumeur dans la France de la Restauration, Paris, Éditions de l'EHESS, 2011.

Mazurel, Hervé, Vertiges de la guerre. Byron, les philhellènes et le mirage grec, Paris, Les Belles Lettres, 2013.

Mollier, Jean-Yves, Reid, Martine et Yon, Jean-Claude (dir.), Repenser la Restauration, Paris, Nouveau Monde, 2005.

Nagy, Laurent, D’une Terreur à l'autre. Théories du complot et nostalgie de l'Empire, 18151816, Paris, Vendémiaire, 2012.

Parent-Lardeur, Françoise, Lire à Paris au temps de Balzac. Les cabinets de lecture à Paris, 1815-1830, Paris, Éditions de l'EHESS, 1981.

Petiteau, Natalie, Lendemains d'Empire. Les soldats de Napoléon dans la France du XIXe siècle, Paris, La Boutique de l'Histoire, 2003.

Petiteau, Natalie, Écrire la mémoire. Les mémorialistes de la Révolution et de l'Empire, Paris, Les Indes savantes / La Boutique de l'histoire, 2012.

Picon, Antoine, Les saint-simoniens. Raison, imaginaire et utopie, Paris, Belin, 2002.

Ploux, François, Guerres paysannes en Quercy. Violences, conciliations et répression pénale dans les campagnes du Lot (1810-1860), Paris, La Boutique de l'Histoire, 2002.

Ploux, François, De bouche à oreille. Naissance et propagation des rumeurs dans la France du XIXe siècle, Paris, Aubier, 2003.

Robert, Vincent, Le temps des banquets. Politique et symbolique d’une génération (18181848), Paris, Publications de la Sorbonne, 2010.

Roquette, Antoine, Le Concordat de 1817. Louis XVIII face à Pie VII, Paris, Le Félin, 2010. 
Rosanvallon, Pierre, Le Sacre du citoyen. Histoire du suffrage universel en France, Paris, Gallimard, 1992.

Rosanvallon, Pierre, La Monarchie impossible. Histoire des Chartes de 1814 et 1830, Paris, Fayard, 1994.

Rosanvallon, Pierre, Le Peuple introuvable. Histoire de la représentation démocratique en France, Paris, Gallimard, 1998.

Rosanvallon, Pierre, La Démocratie inachevée. Histoire de la souveraineté du peuple en France, Paris, Gallimard, 2000.

Vidalenc, Jean, La Restauration, Paris, PUF, 1966, coll. Que sais-Je?

Voilliot, Christophe, La Candidature officielle. Une pratique d'État de la Restauration à la Troisième République, Rennes, PUR, 2005.

Waquet, Françoise, Les Fêtes royales sous la Restauration ou l'Ancien régime retrouvé, Paris-Genève, Droz, 1981.

Waresquiel, Emmanuel de et Yvert, Benoît, Histoire de la Restauration, 1814-1830. Naissance de la France moderne, Paris, Perrin, 1996.

Waresquiel, Emmanuel de, L'Histoire à rebrousse-poil. Les élites, la Restauration, la Révolution, Paris, Fayard, 2005.

Waresquiel, Emmanuel de, Un groupe d'hommes considérables. Les pairs de France et la Chambre des pairs héréditaire de la Restauration, 1814-1831, Paris, Fayard, 2006.

Zanone, Damien, Écrire son temps. Les Mémoires en France de 1815 à 1848, Lyon, Presses Universitaires de Lyon, 2006. 